\title{
5-ALA ameliorates hepatic steatosis through AMPK signaling pathway
}

\author{
Haoyong Yu1,*, Mingliang Zhang1,*, Yunqin Ma1, Junxi Lu¹, Jiemin Pan', Pan Pan', \\ Haibing Chen ${ }^{1,2}$ and Weiping Jia ${ }^{1}$
}

'Department of Endocrinology and Metabolism, Shanghai Jiao Tong University Affiliated Sixth People's Hospital, Shanghai Diabetes Institute, Shanghai Clinical Center of Diabetes, Shanghai Key Laboratory of Diabetes Mellitus, Shanghai Key Clinical Center for Metabolic Disease, Shanghai, China 2Department of Endocrinology and Metabolism, Shanghai Eighth People's Hospital Affiliated to Jiangsu University, Shanghai, China

*(H Yu and M Zhang contributed equally to this work)

\author{
Correspondence \\ should be addressed \\ to $\mathrm{H}$ Chen or W Jia \\ Email \\ 18930173813@163.com or \\ wpjia@sjtu.edu.cn
}

\begin{abstract}
5-Aminolevulinic acid (5-ALA), the first compound in the porphyrin synthesis pathway, has been reported to ameliorate the diabetic state in Otsuka Long-Evans Tokushima Fatty rats by reducing fat pad weight in the retroperitoneal region. Dietary supplementation with 5-ALA has additionally demonstrated the capacity to lower blood glucose and $\mathrm{HbA1c}$ levels among subjects with diabetes. The etiology of nonalcoholic fatty liver disease (NAFLD) is complex and its typical characteristics include obesity and insulin resistance. As 5-ALA supplementation has previously normalized glucose and insulin resistance, we sought to investigate whether 5-ALA had potential therapeutic effects on NAFLD and elucidate the signal pathway mediating these effects. To explore these questions, we fed C57BL/6J mice a high-fat diet (HFD) to induce a fatty liver disease and supplemented the diet-induced obese (DIO) mice with 5-ALA. The mice in the presence of 5-ALA demonstrated a decrease in body weight and hepatic lipid content and moderate improvement in glucose homeostasis compared to untreated controls. Further, we found that 5-ALA activated AMPK signaling pathway, which was correlated with enhanced lipolysis and fatty acid $\beta$-oxidation. Human hepatocarcinoma cells (HepG2 cells) treated with 5-ALA were additionally used to investigate the mechanics of 5-ALA. Treated cells had a higher expression of lipolysis-related genes, including PGC-1 $\alpha$. Our data indicated that 5-ALA might represent a novel compound that could be useful for the treatment of nonalcoholic fatty liver disease (NAFLD), likely through the restoration of phosphorylation levels of AMPK (Thr172) and acetyl-CoA (ACC) (Ser79), further enhanced PGC1 $\alpha$ and CPT1 $\alpha$ expression.
\end{abstract}

\section{Key Words}

-5-aminolevulinic acid

- nonalcoholic fatty liver disease

- AMPK

- obesity
Journal of Molecular

Endocrinology

(2017) 59, 121-128

\section{Introduction}

NAFLD is the most common liver disease worldwide. It comprises a spectrum of disorders characterized by liver steatosis, with $>5 \%$ of hepatocytes infiltrated by fat in individuals with no history of alcohol abuse or competing etiologies for hepatic steatosis (Mavrogiannaki \& Migdalis 2013). Frequently, NAFLD clusters with metabolic abnormalities, including type 2 diabetes mellitus (T2DM), obesity, hypertension, hyperlipidemia, etc. Growing http://jme.endocrinology-journals.org DOI: 10.1530/JME-16-0260
(C) 2017 Society for Endocrinology Printed in Great Britain
Published by Bioscientifica Ltd 
evidence has suggested that the main characteristic of NAFLD include insulin resistance (Fracanzani et al. 2008), increased free fatty acids reflux from adipose tissue to the liver, hepatocyte lipotoxicity (Malhi \& Gores 2008), cardiovascular disease and aortic valve sclerosis (Targher et al. 2010). To date, no optimal treatment has been found, underscoring the need for further efforts in elucidating the pathogenesis of NAFLD and distinguishing effective pharmacological therapies.

5-ALA, a naturally occurring precursor of porphyrin, is widely distributed in both plant and animal cells and serves as the biological precursor in the heme biosynthetic pathway. As the precursor of heme, ALA is an essential molecule in human and other vertebrates and may be associated with various metabolic disorders. Indeed, it has been reported that 5-ALA ameliorated the diabetic state more effectively than metformin in Otsuka Long-Evans Tokushima Fatty rats by reducing fat pad weight in the retroperitoneal region through a decrease in adipocyte mitochondrial content (Sato et al. 2014). This suggests the usefulness of this compound as an antidiabetic agent. Some clinical studies have further suggested that 5-ALA can improve the glucose profile of prediabetic subjects (Rodriguez et al. 2012). As a typical characteristic of NAFLD is insulin resistance, we hypothesized that 5-ALA supplementation could improve insulin resistance and set out to investigate the beneficial effects on hepatic lipid metabolism and NAFLD, which to date have remained unclear.

AMPK is a member of a larger metabolite-sensing protein kinase family and a highly conserved heterotrimeric serine/threonine protein kinase that regulates energy homeostasis in mammalian cells. AMPK activated by adenosine triphosphate (ATP) depletion or adenosine monophosphate (AMP) elevation can drive multiple cellular signaling pathways and exert diverse effects on lipid metabolism (Seo et al. 2015). AMPK regulates both the fatty acid oxidation by the phosphorylation of acetyl-CoA carboxylase (ACC) (Hawley et al. 1996) and mitochondrial biogenesis through the increase of the expression of vital proteins for proper mitochondrial function, including peroxisome proliferator activated receptor c coactivator $1 \alpha$ (PGC-1 $\alpha$ ) (Baar et al. 2002) and carnitine palmitoyl transferase $1 \alpha(\mathrm{CPT} 1 \alpha)$. AMPK activation increases PGC$1 \alpha$ expression, which is a key downstream mediator of the effects of AMPK on mitochondrial function and fatty acid $\beta$-oxidation (Baar et al. 2002, Handschin \& Spiegelman 2006). CPT $1 \alpha$ is responsible for the transport of long-chain fatty acids into the mitochondria along with carnitine, to enhance fatty acid oxidation (Bruce et al. 2009). Impaired AMPK activation is associated with intracellular lipid metabolism disorders and strategies that activate AMPK and downstream PGC- $1 \alpha$ and CPT $1 \alpha$ can be harnessed for the prevention and treatment of lipid metabolism disorders (Ruderman et al. 2013). A previous study (Ji et al. 2010) has indicated that 5-ALA mediated photodynamic therapy via the AMPK pathway. On this basis, this study was designed to investigate the metabolism-regulating effects and related mechanism of 5-ALA in NAFLD.

\section{Materials and methods}

\section{Animals and diet}

Laboratory Animals, Male C57BL/6J mice were purchased from Shanghai SLAC Laboratory Animal Co. Ltd. Mice were housed in laboratory cages at a temperature of $23 \pm 3^{\circ} \mathrm{C}$ with a humidity of $35 \pm 5 \%$ under a 12 -h darkness/ light cycle (lights on/off at 07:00 h/19:00 h). Animals were maintained on a regular chow diet (Shanghai Laboratory Animal Co. Ltd, Shanghai, China). For the diet-induced obesity model, mice at 8 weeks of age were fed a highfat diet (HFD) containing 60\% kcal of fat (D12492; Research Diets, Inc., NJ, USA) for 18 weeks. 5-ALA hydrochloride (catalog number: 20130701) was provided by Tian Yi Bio Co., Ltd. (Suzhou, China).

\section{Animal treatments}

All experimental procedures were approved by the Shanghai Jiao Tong University Experimental Animal Ethics Committee. The mice had free access to feed and distilled water. DIO animals were randomized into 5-ALA treatment groups ( $n=5)$ and HFD control group $(n=5)$. After 18 weeks of HFD diets, 5-ALA was administered as a solution in phosphate buffer saline (PBS), $5 \mathrm{mg} / \mathrm{kg}$, orally once a day or PBS as vehicle for 10 weeks in high-fat diet-induced obese mice.

\section{Cell culture and treatments}

Human hepatocarcinoma (HepG2) cells were maintained in Dulbecco's modification of Eagle's medium (DMEM) containing $10 \%$ fatal bovine serum at $37^{\circ} \mathrm{C}, 5 \% \mathrm{CO}_{2}$. All culture media were supplemented with penicillin $(100 \mathrm{U} / \mathrm{mL})$ and streptomycin $(100 \mu \mathrm{g})$. 5-ALA was dissolved in PBS and diluted in DMEM to a final concentration of $200 \mu \mathrm{M}$. HepG2 cells were incubated with 5-ALA for $15 \mathrm{~min}$, $30 \mathrm{~min}, 2 \mathrm{~h}$ or $4 \mathrm{~h}$. Another group of HepG2 cells were incubated with $100 \mu \mathrm{M}$ 5-aminoimidazole-4-carboxamide 1 - $\beta$-D-ribofuranoside (AICAR) for $30 \mathrm{~min}$ as a positive control.

Published by Bioscientifica Ltd. 


\section{Body weight, food intake and fat mass}

Throughout the experimental period, body weight and food intake were assessed weekly. After 10 weeks of treatment, animals were then killed by $2 \%$ pentobarbital sodium and necropsied, and liver was excised immediately, weighed and taken for estimation of triglyceride content. Different adipose tissues were separated and weighed, while livers were snap-frozen in liquid nitrogen immediately after resection before being stored at $-80^{\circ} \mathrm{C}$.

\section{Glucose homeostasis}

Blood samples were collected by the tail snipping method after overnight fasting (12h) to measure fasting glucose levels using the ACCU-CHEK glucometer (Roche diagnostics) once a week during the entire duration of study. Insulin tolerance tests (ITT) were performed on the 8 th week of treatment. Before ITT, mice were fasted for $6 \mathrm{~h}$ before being weighed. Blood samples were collected from the tail vein to evaluate fasting plasma glucose levels. Next, animals received an intraperitoneal injection of regular human insulin at $0.75 \mathrm{U} / \mathrm{kg}$ body weight (Humulin regular insulin, Eli Lily). Serum glucose levels were subsequently measured at 0, 15, 30, 60 and $90 \mathrm{~min}$ after insulin injection using the ACCU-CHEK glucometer.

Glucose tolerance test (GTT) was performed on the 9 th week of treatment $0,30,60$, and $90 \mathrm{~min}$ after $1 \mathrm{~g} / \mathrm{kg}$ glucose challenge using ACCU-CHEK glucometer by tail clip method.

\section{Histological and morphometric analysis of liver}

Liver tissue was isolated and fixed with $4 \%$ paraformaldehyde for 24-30 h at room temperature and embedded in paraffin. $5 \mu \mathrm{m}$ thick paraffin-embedded tissue sections were then deparaffinized and rehydrated in graduated alcohol in distilled water. Digital images of hepatic tissue sections were captured using a light microscope (Olympus) at $\times 10$ and $\times 20$ magnification. For each group, 5 fields from each of the 5 different hematoxylin-eosin stained sections per animal were analyzed.

\section{Measurement of hepatic triglyceride (TG) content}

Triglyceride (TG) contents in mice were measured using the triglyceride determination kit (Sigma, triglyceride reagent T2449 and free glycerol reagent F6428) according to the manufacturer's instructions. After extraction of total liver lipids with chloroform/methanol (2:1, vol/vol), the dried lipid was re-suspended in $1 \mathrm{~mL}$ of $100 \%$ ethanol and optical density (OD) value was determined at $540 \mathrm{~nm}$. The TG content was expressed in milligrams of lipid per gram of liver tissue.

\section{Real-time quantitative polymerase chain reactions (RT- PCR) analysis}

Total RNA was extracted from of each mouse liver sample and cultured HepG2 cells by TRIzol reagent (Invitrogen Biotechnologies) according to the manufacturer's instructions. For reverse transcription, $1 \mu \mathrm{g}$ of the total mRNA was converted to first-strand complementary DNA in $20 \mu \mathrm{L}$ reactions by Moloney murine leukemia virus-reverse transcriptase using random hexamer primers (Invitrogen) with a cDNA synthesis kit (Promega). Quantitative RT-PCR was conducted with an ABI Prism 7500 sequence detection system, using the SYBR Green PCR Master Mix (Applied Biosystems). The primer sequences are listed as follows and 18s expression was quantified and used as an internal control for normalization according to the $2-\Delta \Delta$ cycle threshold method. The oligonucleotides used for RT-PCR were synthesized by JieLi Biotechnology Co. (Shanghai) as follows:

PGC-1 $\alpha$ (h) Forward 5'-CCTCAGTCCCAGTCGCATTC-3',

Reverse 5'-TTCTCATAGTCGCGTCGGTACTC-3';

PPAR $\alpha$ (h) Forward 5'-GGGATGCTGGTAGCGTATGG-3',

Reverse 5'-AACTTCATGGCAAAATCAAACTTG-3';

CPT1 $\alpha$ (h) Forward 5'-GATTTTGCTGTCGGTCTTGG-3',

Reverse 5'-CTCTTGCTGCCTGAATGTGA-3';

Actin (h) Forward 5'-AAGGAGCCCCACGAGAAAAAT-3', Reverse 5'-ACCGAACTTGCATTGATTCCAG3';

Glucose-6-phosphatase (m) Forward

5'-CACAGTGGACGACATCCGAAA-3',

Reverse 5'-GCAGGGCATTCATGTGGCT3';

PEPCK (m) Forward 5'-CTGCATAACGGTCTGGACTTC-3',

Reverse 5'-GCCTTCCACGAACTTCCTCAC3';

PGC- $1 \alpha(\mathrm{m})$ Forward

5'-AGAACGTGACCTTATCACCCC-3',

Reverse 5'-GCACCTCAACCCGACTACTT3';

CPT1 $\alpha(\mathrm{m})$ Forward

5'-CTCAGTGGGAGCGACTCTTCA-3', Reverse 5'-GGCCTCTGTGGTACACGACAA3';

Actin (m) Forward 5'-ATGACCCAAGCCGAGAAGG-3', Reverse 5'-CGGCCAAGTCTTAGAGTTGTTG3'. http://jme.endocrinology-journals.org DOI: 10.1530/JME-16-0260
() 2017 Society for Endocrinology Printed in Great Britain
Published by Bioscientifica Ltd 


\section{Western blot analysis}

We extracted proteins from mouse livers and cultured HepG2 cells by RIPA lysis buffer $(150 \mathrm{mM} \mathrm{NaCl}, 1 \%$ Nonidet P-40, 0.5\% sodium deoxycholate, 0.1\% SDS, $50 \mathrm{mM}$ Tris- $\mathrm{HCl}, \mathrm{pH} 7.4)$ with the protease inhibitor phenylmethanesulfonyl fluoride (PMSF). The protein content was assayed by BCA protein assay reagent (Pierce) according to the manufacturer's protocol. Protein lysates were further resolved by SDS-PAGE and then transferred to a nitrocellulose membrane. We blocked the nitrocellulose membrane with Tris-buffered saline with Tween 20 containing 5\% nonfat milk, followed by incubation with the following primary antibodies overnight at $4^{\circ} \mathrm{C}$ : phosphor-AMPK, AMPK, acetyl-CoA (ACC), phosphor-ACC, CPT $1 \alpha$, PGC- $1 \alpha$ and heat shock proteins (HSP90). All the above antibodies were purchased from Cell Signaling Technology. Blots were then probed with corresponding horseradish peroxidase-conjugated secondary antibodies (Cell Signaling) and visualized by an enhanced chemiluminescence detection kit. All the protein levels were normalized to HSP90 expression and expressed as arbitrary units.

\section{Primary hepatocyte isolation}

Primary hepatocytes were isolated from AMPK $\alpha 1 /$ AMPK $\alpha 2$ flox (gift from Prof. Li Jia, Shanghai Institue of Materia Medica) and control mice, which have been fasted for $2 \mathrm{~h}$. After isolated cells were plated on collagen type I-coated 12-well plates in DMEM with 10\% fetal bovine serum at a density of $5 \times 10^{5}$ cells per well. After $12 \mathrm{~h}$, hepatocytes transfected with adenovirus-cre, and then after $24 \mathrm{~h}$, primary hepatocytes incubated with 5-ALA $(300 \mu \mathrm{m})$ for $4 \mathrm{~h}$ to detect the protein expression.

\section{Statistical analysis}

All statistical analyses were performed with Prism Software (Graph Pad) using the independent $t$-test. Data are presented as mean \pm S.E.M., with $P<0.05$ considered as statistically significant.

\section{Results}

\section{5-ALA decreased body weight and improved glucose tolerance in diet-induced obese mice}

We conducted 5-ALA dietary supplementation experiments to analyze its phenotypic effects on DIO mice over the course of 10 weeks. 5-ALA administration resulted in a gradual body weight decrease from the first treatment week, reaching a maximum decrease of $16 \%$ body weight on the 10th week of treatment (Fig. 1A and B). During the course of the study, food consumption was recorded weekly and did not show a significant difference between the 5-ALA-treated group and the vehicle-treated control HFD mice (Fig. 1C).

We compared glucose tolerance among the two groups of mice to examine whether 5-ALA correlated with glucose metabolism. 5-ALA-treated mice exhibited lower blood glucose levels compared to vehicle-treated mice beginning the 7 th week of treatment (Fig. 1D). We further tested the impact of 5-ALA on glucose intolerance in both groups. The data indicated an improvement in glucose intolerance in the 5-ALA group as measured by an intraperitoneal GTT. We observed $\sim 10 \%$ decrease in glucose excursion as compared to controls (glucose AUC of 5-ALA treated vs vehicle treated; $P<0.05$ ) (Fig. 1E). Compared to vehicle-treated animals, 5-ALA-treated mice also demonstrated a significant decrease in serum glucose concentrations according to an ITT (Fig. 1F).

\section{5-ALA decreased white adipose tissue content and relieved hepatic steatosis}

At the end of the study, fresh liver and surrounding epididymal fat tissue were weighed. Significant decreases in the weight of the two tissues were observed in the 5-ALAtreated group compared to the vehicle-treated group (Fig. 2A and B). TG levels were also decreased by 5-ALA supplementation compared to controls $(43.75 \pm 4.03$ vs $29 \pm 2.58 \mathrm{mg} / \mathrm{g}$ tissue; $P<0.05$ ) (Fig. 2C). Histological evaluation of liver from the two groups also demonstrated that HFD animals had increased macro-vesicular steatosis with larger lipid droplets compared to lean controls. In 5-ALA-treated animals, a significant reduction in macrovesicular steatosis was observed (Fig. 2D), which was consistent with the decrease in hepatic TG content.

\section{5-ALA promoted lipolysis and fatty acid beta-oxidation to ameliorate hepatic steatosis through AMPK pathway activation}

5-ALA alleviated hepatic steatosis without exerting changes in the feed consumption during the study period. This may be due to increase in energy expenditure, such as adipose lipolysis and fat oxidation. To investigate this, we compared the gene expression of lipolysis and fatty acid beta-oxidation marker genes including PGC-1 $\alpha$ (Fig. 3A) and CPT1 $\alpha$ (Fig. 3B) among the two groups. The two genes

Published by Bioscientifica Ltd 
A

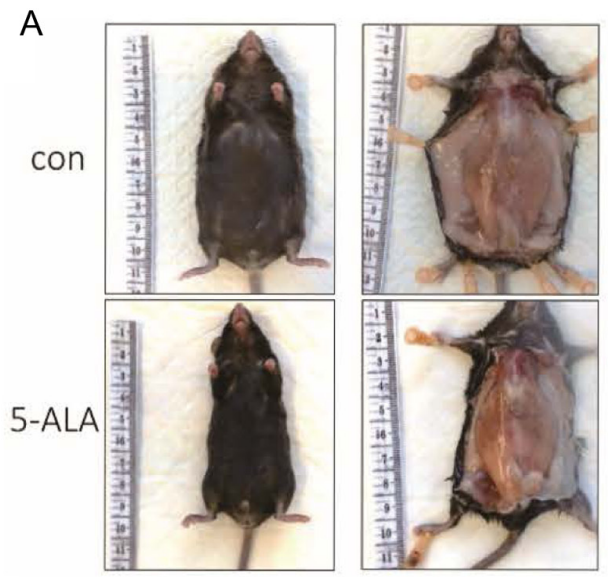

C
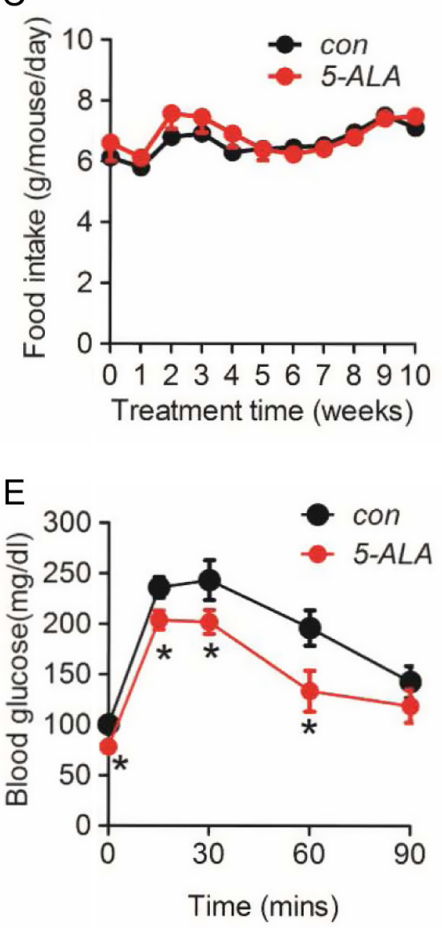

B
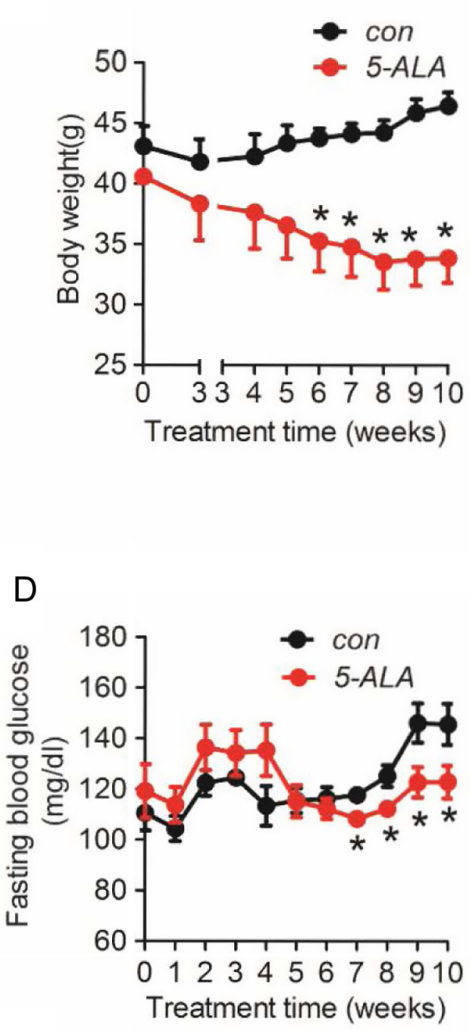

$\mathrm{F}$

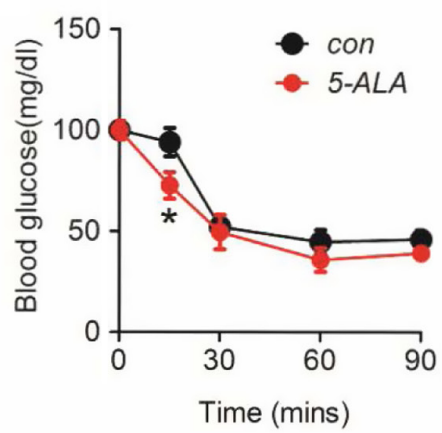

Figure 1

5-ALA decreases body weight and fasting blood glucose of DIO (diet-induced obese) mice. (A and B) Effect of 5-ALA (5-aminolevulinic acid) on body weight. (C) Effect of 5-ALA on food intake. (D) Fasting blood glucose was monitored weekly. (E) Glucose tolerance test in HFD-induced obese mice at week 8 of treatment. (F) Insulin tolerance test in DIO mice at week 8 of treatment. All data are presented as mean \pm S.E.M. $(n=5) . * P<0.05$ vs vehicle-treated group. were increased in the 5-ALA-treated group compared to vehicle-treated animals $(P<0.05)$. The AMPK signaling pathway could enhance energy expenditure through upregulation of adipose lipolysis and fat oxidation, so we investigated whether 5-ALA may activate AMPK signaling through the examination of AMPK pathway proteins. Western blotting results indicated that phosphorylation of AMPK and its downstream marker ACC were increased along with PGC-1 $\alpha$ and CPT1 $\alpha$ expression in the 5-ALA-treated group (Fig. 3C). Additionally, we tested lipogenesis-related genes ACC, SREBP-1C and FAS, though no differences between the two groups were observed (data not shown). These findings implied that 5-ALA may alleviate hepatic steatosis through the AMPK signaling to increase fatty acid oxidation.

\section{5-ALA promoted lipolysis and fatty acid beta-oxidation gene expression and activation of the AMPK pathway in HepG2 cells}

To examine recapitulate and more precisely examine the effects of 5-ALA on lipid metabolism in vitro, we incubated HepG2 cells with $200 \mu \mathrm{m}$ 5-ALA for different time periods (15 min, $30 \mathrm{~min}, 2 \mathrm{~h}, 4 \mathrm{~h}$ ). Western blotting showed that the activation of the AMPK pathway was dependent on the timing of 5-ALA administration, with maximum 


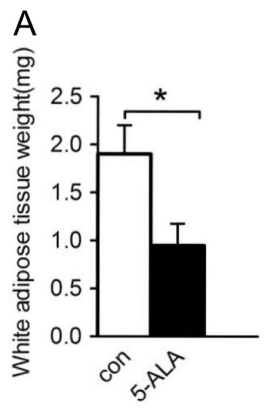

B

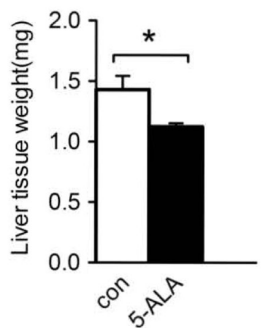

C

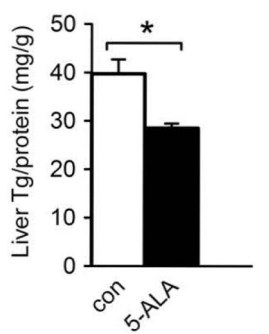

D

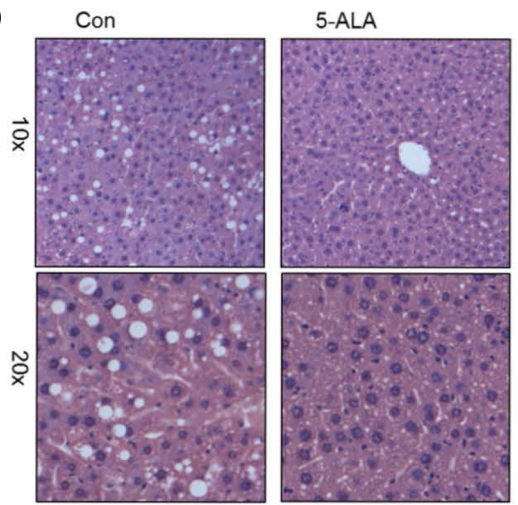

Figure 2

5-ALA reduced fat mass and liver steatosis in diet-induced obese mice. (A) Weight of white adipose tissue measured in two groups. (B) Weight of liver measured in two groups and (C) liver triglyceride measured in two groups. (D) Triglycerides in liver measured by hematoxylin-eosin staining. All data are presented as mean \pm S.E.M. $(n=3) .{ }^{*} P<0.05$ vs vehicle-treated group.

activation occurring $15 \mathrm{~min}$ after incubation (Fig. 4C). 5-ALA activation of AMPK was also dose dependent, achieving optimal activation at a dose of $200 \mu \mathrm{m}$ (figure not shown). As an AMPK activator, AICAR appeared to enhance PGC-1 $\alpha$ (Fig. 4A) and CPT1 $\alpha$ (Fig. 4B) mRNA levels in a quantitative (q) RT-PCR analysis. Consistent with the results of AICAR (taken as a positive control of AMPK activation), 5-ALA also significantly upregulated genes involved in fatty acid oxidation, including PGC- $1 \alpha$ (Fig. 4A) and CPT1 $\alpha$ (Fig. 4B) mRNA levels. Further, Western blotting results showed that phosphorylation of AMPK and its downstream marker ACC increased in tandem with PGC- $1 \alpha$ and CPT1- $\alpha$ expression in HepG2 cells treated with 5-ALA. These results were in accordance with the observed results of 5-ALA-treated animals.

\section{Deletion of hepatic AMPK results in decreased} PGC-1 $\alpha$ and CPT1- $\alpha$ To evaluate the role of hepatic AMPK in 5-ALA action, we deleted AMPK $\alpha 1 /$ AMPK $\alpha 2$ in the primary hepatocytes, which transfected with the Cre recombinase. We have obtained the AMPK $\alpha 1 /$ AMPK $\alpha 2$ flox mice, and isolated the primary hepatocytes, which transfected with the adenovirus-cre for $24 \mathrm{~h}$, and then incubated with 5-ALA, we found that CPT1 $\alpha$ and PGC1 $\alpha$ decreased in primary hepatocytes of AMPK $\alpha 1 /$ AMPK $\alpha 2$ flox mice, compared to the hepatocytes from the control mice (Fig. 5). Therefore, loss of function of AMPK is very critical for the role of 5-ALA.

\section{Discussion}

5-ALA is produced in the heme biosynthetic pathway with no toxic side effects when used as a dietary supplement, according to a report by Rodriguez and coworkers (Rodriguez et al. 2012). A previous report has also shown
A
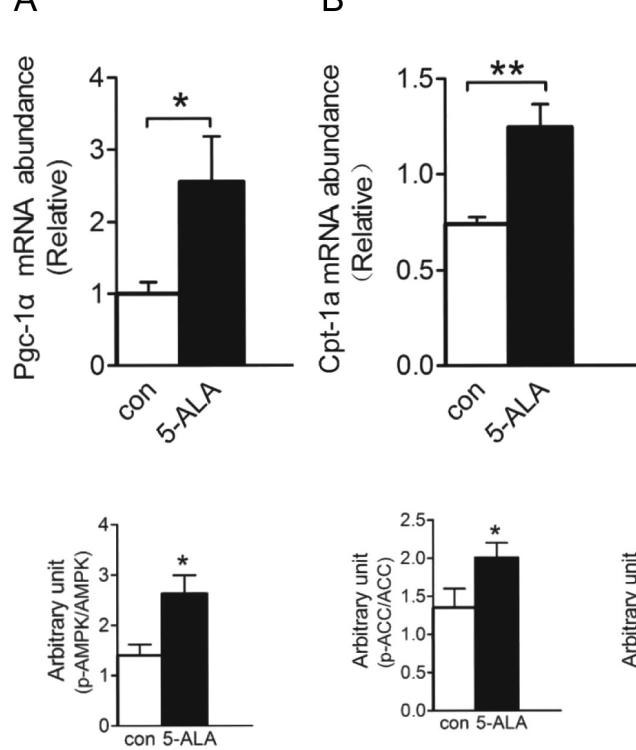

B

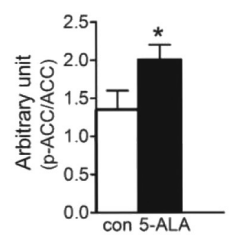

C
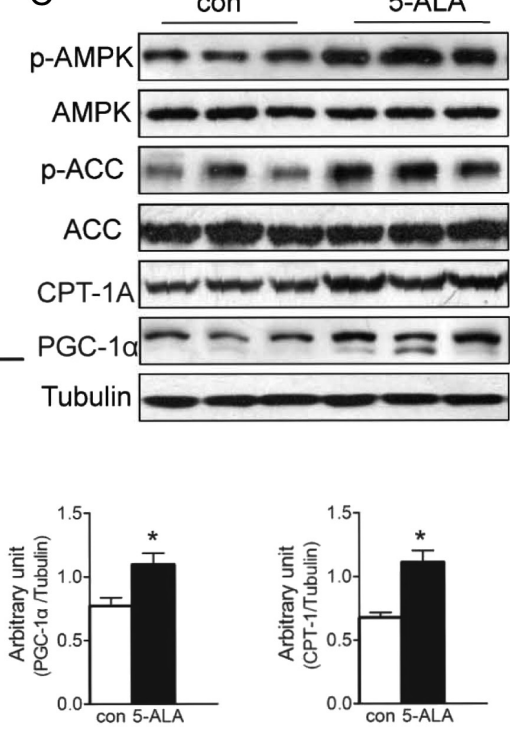

C 2017 Society for Endocrinology Printed in Great Britain

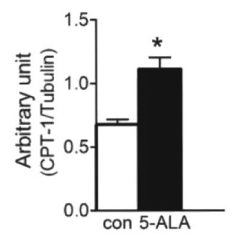

Figure 3

5-ALA activates phosphorylation of AMPK and promotes gene expression of fatty acid betaoxidation in DIO mice. (A) Effect of 5-ALA on PGC- $1 \alpha$ mRNA expression in DIO mice. (B) Effect of 5-ALA on CPT $1 \alpha$ mRNA expression in DIO mice. (C) Phospho-AMPK, phospho-ACC, PGC-1 $\alpha$ and CPT $1 \alpha$ levels in liver measured at week 8 of treatment. All data are presented as mean \pm s.E.M. $(n=5) .{ }^{*} P<0.05$ vs vehicle-treated group. 
A

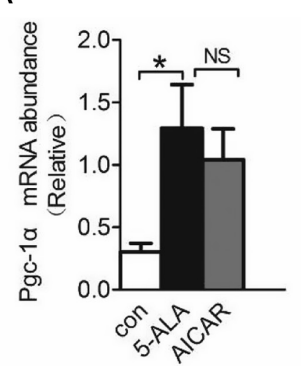

B

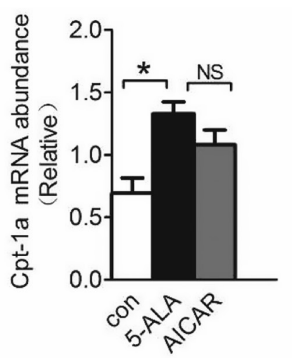

C

\begin{tabular}{|c|c|}
\hline \multirow[b]{2}{*}{ p-AMPK } & $5 \mathrm{~min} 3$ \\
\hline & $-\infty$ \\
\hline AMPK & 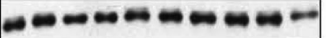 \\
\hline p-ACC & $-x-\infty-\infty$ \\
\hline $\mathrm{ACC}$ & 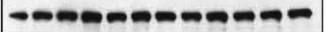 \\
\hline HS & 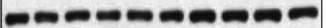 \\
\hline
\end{tabular}

D
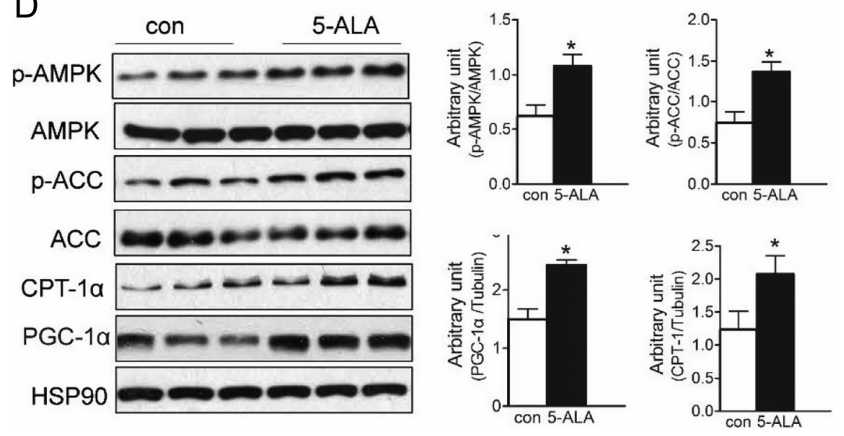

Figure 4

5-ALA activates phosphorylation of AMPK and promotes gene expression of fatty acid beta-oxidation in HepG2 cells. (A and B) Effect of 5-ALA on PGC-1 $\alpha$ and CPT $1 \alpha$ mRNA expression in DIO mice. (C) Phospho-AMPK and phosphoACC levels detected during a time-course. (D) Phospho-AMPK, phospho-ACC, PGC-1 $\alpha$ and CPT1- $\alpha$ levels after ALA treatment in hepG2 cells. All data are presented as mean \pm S.E.M. $(n=5) .{ }^{*} P<0.05$ vs vehicle-treated group.

that 5-ALA could promote aerobic energy metabolism by enhancing Cox activity in mitochondria to increase ATP levels (Ogura et al. 2011), a feature which is supported by the phenotype of the mice in our study. In addition, a previous study revealed that 5-ALA ameliorated diabetic abnormalities in Otsuka Long-Evans Tokushima Fatty rats by reducing the visceral fat mass (Sato et al. 2014). As obesity is a critical risk factor for the development of NAFLD, and strengthening energy expenditure may be an effective approach against NAFLD, we speculated that the lipid-lowering action of 5-ALA may have beneficial effects on the development of NAFLD.

Adipose tissue is responsible for $60 \%$ of $\mathrm{TG}$ accumulation. Excess lipid spill over from the incompetent adipose tissue may lead to lipid deposition in organs such as liver and muscle. Moreover, adipose tissue is a major

source of free fatty acids, hormones, cytokines and other factors secreted into the circulation and frequently taken up by the liver. The peripheral free fatty acid flux derived from visceral adipose tissue is thus a major determinant in the accumulation of hepatic and lipoprotein fat in NAFLD. Identification of a novel molecule or compound that accelerates lipolysis or lipid beta-oxidation is an important pharmacological approach for NAFLD treatment. For the first time here, we put forward that 5-ALA has powerful lipid-lowering capacity that ameliorates hepatic steatosis through the activation of the AMPK signaling pathway.

Our study was designed to investigate the metabolismregulating effects and related mechanisms of 5-ALA. 5-ALA was administered to DIO mice on HFD. Metabolicrelated parameters were measured weekly, including food intake, body weight and fasting blood glucose during the period of 5-ALA treatment. On the 9th week of treatment, we performed ITTs and GTTs to validate 5-ALA's effects on glucose metabolism. After 10 weeks of treatment, animals were killed and liver and adipose tissue were collected for further analysis. In our study, 5-ALA showed weight and hepatic lipid-lowering effects on HFD administered mice without interfering with appetite. 5-ALA suppressed hepatic steatosis, lowered serum triglyceride (TG) and decreased body weight gain in animals with hepatic steatosis. Further, glucose intolerance and hypertriglyceridemia were improved, demonstrating the ability of 5-ALA to ameliorate diabetic abnormalities through the reduction of fat mass. In further support of

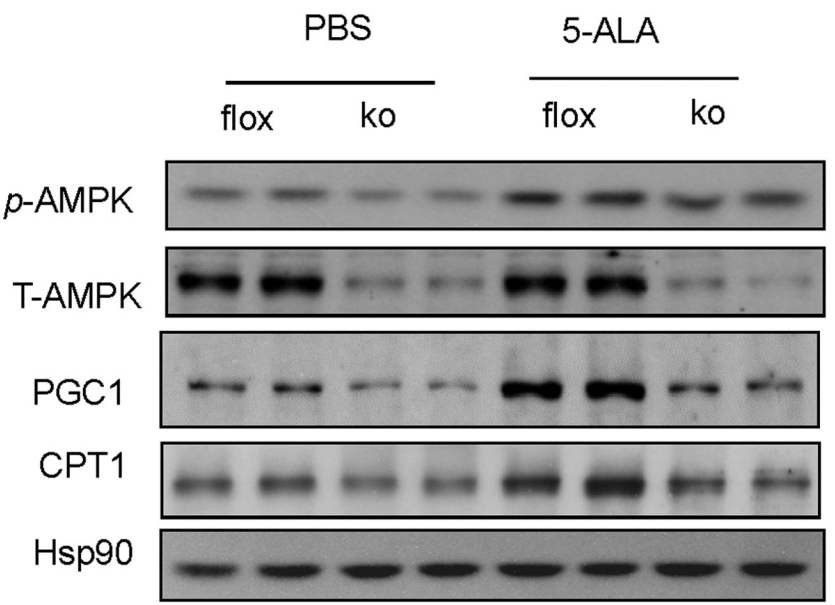

Figure 5

5-ALA increased PGC-1 $\alpha$ and CPT $1 \alpha$ expression through AMPK phosphorylation in mouse primary hepatocytes. Western blot for phospho-AMPK, AMPK, PGC-1 $\alpha$ and CPT1 $\alpha$, ko: primary hepatocytes from AMPK $\alpha 1 /$ AMPK $\alpha 2$ floxed mice transfected with adenovirus-cre. Flox: primary hepatocytes from AMPK $\alpha 1 /$ AMPK $\alpha 2$ floxed mice as a control.

Published by Bioscientifica Ltd. 
this, lipolysis and lipogenesis gene expression patterns were verified in both mouse liver tissue and in a hepatic cell line treated with 5-ALA. Both the in vivo and in vitro assays showed that 5-ALA promoted mitochondrial function and fatty acid $\beta$-oxidation through the activation of AMPK signaling, subsequently modulating the downstream target genes PGC- $1 \alpha$ and CPT1 $\alpha$. 5-ALA also promoted the phosphorylation of AMPK at threonine 170 and upregulated hepatic expression of its target genes involved in fatty acid oxidation (such as hepatic CPT $1 \alpha$ and PGC-1 $\alpha$ ). 5-ALA activated AMPK suppressed hepatic steatosis, lowered serum triglyceride (TG) and decreased body weight gain in animals with hepatic steatosis.

In summary, our results demonstrated that 5-ALA accelerated lipid beta-oxidation to ameliorate hepatic steatosis through the activation of AMPK signaling and upregulation of hepatic expression of its target genes involved in fatty acid oxidation, such as hepatic CPT $1 \alpha$ and PGC- $1 \alpha$. We demonstrated this mechanism not only in 5-ALA-treated DIO mice but also in 5-ALA-treated cultured hepatic human cells, allowing us enhanced confidence in this novel mechanism of 5-ALA action on NAFLD etiology. Most importantly, our findings support the potential therapeutic applications of 5-ALA in the treatment of NAFLD.

\section{Declaration of interest}

The authors have no commercial associations that might be a conflict of interest in relation to this article.

\section{Funding}

This work did not receive any specific grant from any funding agency in the public, commercial, or not-for-profit sector.

\section{Acknowledgments}

This study was supported by grants from National Key Research and Development Project of China (2016YFA0502003), Key Program of the Shanghai Municipality for Basic Research (11JC1409600), 973 Program (2011CB504001), The Innovation Foundation of Translational Medicine of Shanghai Jiao Tong University School of Medicine, the Shanghai SJTUSM Biobank, National Major Scientific and Technological Special Project for 'Significant New Drugs Development' (2011ZX09307-001-02), International Science \& Technology Cooperation Program of China (81170760), National Natural Science Foundation of China (2014DFA31870, 11302130, 81500625) and Municipal Natural Science Foundation of Shanghai (17ZR1421200).

\section{References}

Baar K, Wende AR, Jones TE, Marison M, Nolte LA, Chen M, Kelly DP \& Holloszy JO 2002 Adaptations of skeletal muscle to exercise: rapid increase in the transcriptional coactivator PGC-1. FASEB Journal 16 1879-1886. (doi:10.1096/fj.02-0367com)

Bruce CR, Hoy AJ, Turner N, Watt MJ, Allen TL, Carpenter K, Cooney GJ, Febbraio MA \& Kraegen EW 2009 Overexpression of carnitine palmitoyltransferase- 1 in skeletal muscle is sufficient to enhance fatty acid oxidation and improve high-fat diet-induced insulin resistance. Diabetes 58 550-558. (doi:10.2337/db08-1078)

Fracanzani AL, Valenti L, Bugianesi E, Andreoletti M, Colli A, Vanni E, Bertelli C, Fatta E, Bignamini D, Marchesini G, et al. 2008 Risk of severe liver disease in nonalcoholic fatty liver disease with normal aminotransferase levels: a role for insulin resistance and diabetes. Hepatology 48 792-798. (doi:10.1002/hep.22429)

Handschin C \& Spiegelman BM 2006 Peroxisome proliferator-activated receptor gamma coactivator 1 coactivators, energy homeostasis, and metabolism. Endocrine Reviews 27 728-735. (doi:10.1210/er.2006-0037)

Hawley SA, Davison M, Woods A, Davies SP, Beri RK, Carling D \& Hardie DG 1996 Characterization of the AMP-activated protein kinase kinase from rat liver and identification of threonine 172 as the major site at which it phosphorylates AMP-activated protein kinase. Journal of Biological Chemistry $27127879-27887$. (doi:10.1074/jbc.271.44.27879)

Ji HT, Chien LT, Lin YH, Chien HF \& Chen CT 2010 5-ALA mediated photodynamic therapy induces autophagic cell death via AMP-activated protein kinase. Molecular Cancer 9 91. (doi:10.1186/1476-4598-9-91)

Malhi H \& Gores GJ 2008 Molecular mechanisms of lipotoxicity in nonalcoholic fatty liver disease. Seminars in Liver Disease 28 360-369. (doi:10.1055/s-0028-1091980)

Mavrogiannaki AN \& Migdalis IN 2013 Nonalcoholic fatty liver disease, diabetes mellitus and cardiovascular disease: newer data. International Journal of Endocrinology 2013 450639. (doi:10.1155/2013/450639)

Ogura S, Maruyama K, Hagiya Y, Sugiyama Y, Tsuchiya K, Takahashi K, Abe F, Tabata K, Okura I, Nakajima M, et al. 2011 The effect of 5 -aminolevulinic acid on cytochrome c oxidase activity in mouse liver. BMC Research Notes 4 66. (doi:10.1186/1756-0500-4-66)

Rodriguez BL, Curb JD, Davis J, Shintani T, Perez MH, Apau-Ludlum N, Johnson C \& Harrigan RC 2012 Use of the dietary supplement 5-aminiolevulinic acid (5-ALA) and its relationship with glucose levels and hemoglobin A1C among individuals with prediabetes. Clinical and Translational Science 5 314-320. (doi:10.1111/j.1752-8062.2012.00421.x)

Ruderman NB, Carling D, Prentki M \& Cacicedo JM 2013 AMPK, insulin resistance, and the metabolic syndrome. Journal of Clinical Investigation 123 2764-2772. (doi:10.1172/JCI67227)

Sato T, Yasuzawa T, Uesaka A, Izumi Y, Kamiya A, Tsuchiya K, Kobayashi Y, Kuwahata M \& Kido Y 2014 Type 2 diabetic conditions in Otsuka Long-Evans Tokushima Fatty rats are ameliorated by 5-aminolevulinic acid. Nutrition Research 34 544-551. (doi:10.1016/j. nutres.2014.04.013)

Seo MS, Kim JH, Kim HJ, Chang KC \& Park SW 2015 Honokiol activates the LKB1-AMPK signaling pathway and attenuates the lipid accumulation in hepatocytes. Toxicology and Applied Pharmacology 284 113-124. (doi:10.1016/j.taap.2015.02.020)

Targher G, Day CP \& Bonora E 2010 Risk of cardiovascular disease in patients with nonalcoholic fatty liver disease. New England Journal of Medicine 363 1341-1350. (doi:10.1056/NEJMra0912063)

Received in final form 19 May 2017

Accepted 26 May 2017

Accepted Preprint published online 31 May 2017 http://jme.endocrinology-journals.org

DOI: 10.1530/JME-16-0260
() 2017 Society for Endocrinology Printed in Great Britain
Published by Bioscientifica Ltd 